\title{
Regulation of Human Argininosuccinate Synthetase Gene: Induction by Positive-Acting Nuclear Mechanism in Canavanine-Resistant Cell Variants
}

\author{
Frederick M. Boyce III and Svend O. Freytag \\ Department of Biological Chemistry and Program in Cellular and Molecular Biology, University of Michigan Med- \\ ical School, Ann Arbor, Michigan, 48109-0606
}

Received 21 June 1988-Final 6 September 1988

\begin{abstract}
Nonhepatic human cell variants resistant to the arginine analog, canavanine, express argininosuccinate synthetase $(A S) m R N A$ at levels 200 -fold higher than parental cells without amplification of AS gene sequences. In this report we show that this regulation occurs in the nucleus prior to polyadenylation of $A S$ precursor RNA and occurs through a positive-acting mechanism operating in canavanine-resistant cells. The half-life of cytoplasmic AS mRNA was estimated by blocking cellular transcription with actinomycin $D$. In both parental and canavanine-resistant variants of RPMI 2650 cells, the AS $m R N A$ decayed with a half-life of 12-24 h, showing that cytoplasmic $m R N A$ stabilization was not involved in this regulation. Quantification of $A S R N A$ following cell fractionation showed that AS precursor $R N A$ was present at greatly elevated amounts in the nuclei of canavanine-resistant cells. Similar results were obtained when nonpolyadenylated $R N A$ was examined. Thus, the mechanism underlying high expression of $A S M R N A$ in canavanine-resistant cells is an early nuclear event, and the processes of polyadenylation and transport of RNA to the cytoplasm are not involved. Intraspecific somatic cell hybrids were constructed to test whether the induction of AS $m R N A$ was due to a gain of a function in canavanine-resistant cells or to a loss of a function in parental cells. Quantification of $A S m R N A$ in hybrid cell lines showed that such cells contained high levels similar to those found in the canavanine-resistant parent. These findings show that the induction of $A S m R N A$ is due to a positive-acting mechanism operating in the nucleus of canavanine-resistant cells.
\end{abstract}

\section{INTRODUCTION}

The human argininosuccinate synthetase $(A S)$ gene is subject to two independently acting control mechanisms in cultured cells $(1,2)$. In nonhepatic tissues, where it plays a role in arginine biosynthesis, AS is expressed at relatively low levels as compared to liver. In several nonhepatic cell types, AS is subject to metabolite-mediated repression by arginine $(3,4)$. In previous reports, we (1) and others (2) showed that the bacterial chloramphenicol acetyltransferase $(C A T)$ gene under the transcriptional control of the human AS promoter was subject to regulation by arginine.

The human AS gene is also subject to another control mechanism in cultured cells that results in high expression of AS mRNA. Human cell variants expressing high levels of AS can be selected by growth in the presence of canavanine, a toxic arginine analog. These variants arise at a frequency of about $10^{-6}$ to $10^{-7}$ from the human squamous cell carcinoma line, RPMI 2650, and express the AS 
mRNA at levels 200-fold higher than parental cells and similar to that in highly differentiated cell types such as normal human hepatocytes. This system is unique in that the increased expression of AS mRNA in resistant cells is not due to amplification of $A S$ gene sequences (4). Once established, the canavanine-resistant phenotype is stable, suggesting that a genetic or stable epigenetic event has occurred in the canavanine-resistant cell.

We have studied $A S$ gene expression in canavanine-resistant cell variants, since it is possible that the mechanism used to achieve high AS mRNA levels might be similar (identical) to that used in normal human liver. The canavanine-resistant phenotype is selectable, thus this system provides a means for the cloning of trans-acting loci that activate a liver function (high $A S$ gene expression) using genomic DNA transfection. Such experiments are feasible only if a single locus is involved and the resulting phenotype is dominant, implying a gain of a function. In this report we show that the 200 -fold increased expression of AS mRNA occurs through a dominant, positive-acting mechanism operating in the nucleus of the canavanine-resistant cell.

\section{MATERIALS AND METHODS}

Somatic Cell Hybrids. The parental cell lines used for the construction of the somatic cell hybrids were RP10C4, a previously described G418-resistant RPMI 2650 cell line (1), and $\mathrm{C} 6 \mathrm{C} 2$, a mycophenolic acid-resistant and canavanine-resistant line obtained by transfection of Can ${ }^{\mathrm{r}}-1$ cells (4) with RSVgpt (5). One day prior to fusions, $1 \times 10^{6}$ cells of each parent were seeded into a single $35-\mathrm{mm}$ dish. Fusions were performed by rinsing cell monolayers with phosphate-buffered saline (PBS), addition of $1 \mathrm{ml} 50 \%$ PEG 1500 (polyethylene glycol, Boehringer Mannheim) for 1 min, followed by extensive rinsing with PBS and replacement of normal growth medium
(Dulbecco's modified Eagle's medium, DMEM, supplemented with $10 \%$ fetal calf serum). Two days later cells were detached by trypsinization and seeded into $100-\mathrm{mm}$ dishes containing normal growth medium supple-

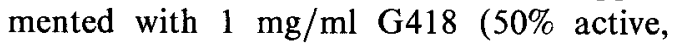
GIBCO), $15 \mu \mathrm{g} / \mathrm{ml}$ hypoxanthine, $2 \mu \mathrm{g} / \mathrm{ml}$ aminopterin, $10 \mu \mathrm{g} / \mathrm{ml}$ thymidine, $25 \mu \mathrm{g} / \mathrm{ml}$ mycophenolic acid, and $250 \mu \mathrm{g} / \mathrm{ml}$ xanthine. Individual colonies were isolated four weeks later using cloning cylinders.

RNA Isolation and S1 Nuclease Assays. Total cellular RNA was isolated using the guanidinium isothiocyanate- $\mathrm{CsCl}$ density gradient method (6). Polyadenylated RNA was isolated by affinity chromatography on oligo-dT cellulose as described (6). Nuclear and cytoplasmic RNAs were prepared from approximately $10^{9}$ cells as described (6). Briefly, cells were harvested in PBS, collected by centrifugation at $2000 \mathrm{~g}$ for $5 \mathrm{~min}$, and lysed by mild vortexing in lysis buffer $(0.14 \mathrm{M}$ $\mathrm{NaCl}, 1.5 \mathrm{mM} \mathrm{MgCl}, 10 \mathrm{mM}$ Tris $\mathrm{HCl}(\mathrm{pH}$ 8.6), $0.5 \%$ Triton $\mathrm{X}-100$, and $100 \mathrm{mM}$ vanadyl ribonucleotide complex). The nuclei were purified by overlay onto lysis buffer containing $0.3 \mathrm{M}$ sucrose followed by centrifugation at $10,000 \mathrm{~g}$ for $20 \mathrm{~min}$. The turbid supernatant containing cytoplasm was deproteinized by digestion with $200 \mu \mathrm{g} / \mathrm{ml}$ proteinase $\mathrm{K}$ for 30 min at $37^{\circ} \mathrm{C}$ and extracted with phenol/ $\mathrm{CHCl}_{3}$. Nuclei were lysed by the addition of SDS to a final concentration of $1 \%$, genomic DNA was sheared by passage through a 21gauge needle, and the extract was deproteinized as described above. DNA was then removed from nuclear samples by digestion with 100 units/ml RQ1 DNase for $30 \mathrm{~min}$ at $37^{\circ} \mathrm{C}$. Oligodeoxynucleotides were removed by precipitation in $20 \%$ sodium acetate. The nuclear RNA was free of ribosomal RNA, and the yield was approximately $10 \%$ of the cytoplasmic yield. S1 nuclease assays were performed essentially as previously described (7), and $\left[{ }^{32} \mathrm{P}\right]$ radioactive probes were prepared using standard procedures. Differences between the intensities of two hybridization 
signals in the autoradiograms were quantified by applying serial dilutions of the more radioactive sample on the same gel.

Actinomycin D Inhibition. Transcription was blocked in subconfluent dishes $(150 \mathrm{~mm})$ of RPMI 2650 and Can $^{\mathrm{r}}-1$ cells (4) by the addition of $20 \mathrm{ml}$ of fresh growth medium containing $10 \mu \mathrm{g} / \mathrm{ml}$ actinomycin D (mannitol salt, Sigma). Cells were harvested at specific times thereafter and total cellular RNA was prepared.

\section{RESULTS}

AS Gene is Weakly Transcribed and $m R N A$ is Very Stable. Previous studies showed that AS promoter-CAT minigenes containing approximately $3 \mathrm{~kb}$ of $\mathrm{AS} 5^{\prime}$ flanking sequence are not more transcriptionally active in canavanine-resistant cells $(1,2)$. Therefore, in order to test for transcriptional regulation more directly, we attempted to measure $A S$ gene transcription rates in RPMI 2650 and $\mathrm{Can}^{\mathrm{r}}-1$ cells using nuclear "run-on" assays $(8,9)$. Although we could readily detect specific transcription of genes that are expressed abundantly in both cell types (actin and phosphoglycerate kinase), we were repeatedly unsuccessful in detecting $A S$ gene transcription using either cDNA or intron probes (data not shown). We were able to demonstrate that in vitro synthesized AS mRNA was detectable in our assays (hybridization efficiencies were $15-50 \%$ ) and that background hybridization was low (less than 5 ppm). Given that the AS mRNA is present at about 3 copies per cell in RPMI 2650 cells and approximately 500 copies per cell in canavanine-resistant cells (unpublished observations) and that the half-life of the AS mRNA is relatively long (see below), it was not surprising to find that the $A S$ gene is weakly transcribed in the cell lines employed in these studies. This is consistent with the idea that the $A S$ gene is considered a "housekeeping" gene in nonhepatic tissue and that transcription of such genes is often below detec- tion in cultured cell lines. For example, transcription of the dihydrofolate reductase gene, another "housekeeping" gene, is detectable only in cell lines in which the gene is amplified 500-fold (10). Since $A S$ gene transcription was not measurable in either cell type, we took a more indirect approach to examine the molecular basis of this regulation.

The degradation rate of mature AS mRNA was estimated by blocking cellular gene transcription with actinomycin $D$, and the decay of specific mRNA was monitored using quantitative $S 1$ nuclease assays (Fig. 1). The data show that the AS mRNA was very stable in both RPMI 2650 and Can ${ }^{\mathrm{r}}-1$ cells with a half-life of approximately $12-24 \mathrm{~h}$. The observed decay of AS mRNA was not exponential in either cell line, which is common for long-lived mRNAs, and probably reflects experimental artifacts when using metabolic inhibitors over extended periods of time. Although the mRNA half-life appears to be slightly longer in the $\mathrm{Can}^{\mathrm{r}}-1$ cell line, this minor difference (threefold) is not nearly enough to account for the 200-fold increased levels of AS mRNA in these cells. Based on these results, we tentatively concluded that the increase in AS mRNA in canavanineresistant cells was not the result of cytoplasmic mRNA stability. This conclusion was substantiated by cellular fractionation studies (see below). Although total cellular RNA was used in this experiment, the majority of the cellular AS RNA is mature cytoplasmic mRNA (see fractionation experiments below). Thus, this experiment measures only the decay rate of mature cytoplasmic mRNA and does not exclude a nuclear RNA stabilization or transport effect.

Elevation of Argininosuccinate Synthetase Precursor RNA Levels in CanavanineResistant Cell Nuclei. The mRNA decay experiments described above suggested that the induction of AS mRNA in canavanineresistant cell variants was being controlled in the nucleus. To test for this directly, RPMI 2650 and $\mathrm{Can}^{\mathrm{r}}-1$ cells were fractionated into 


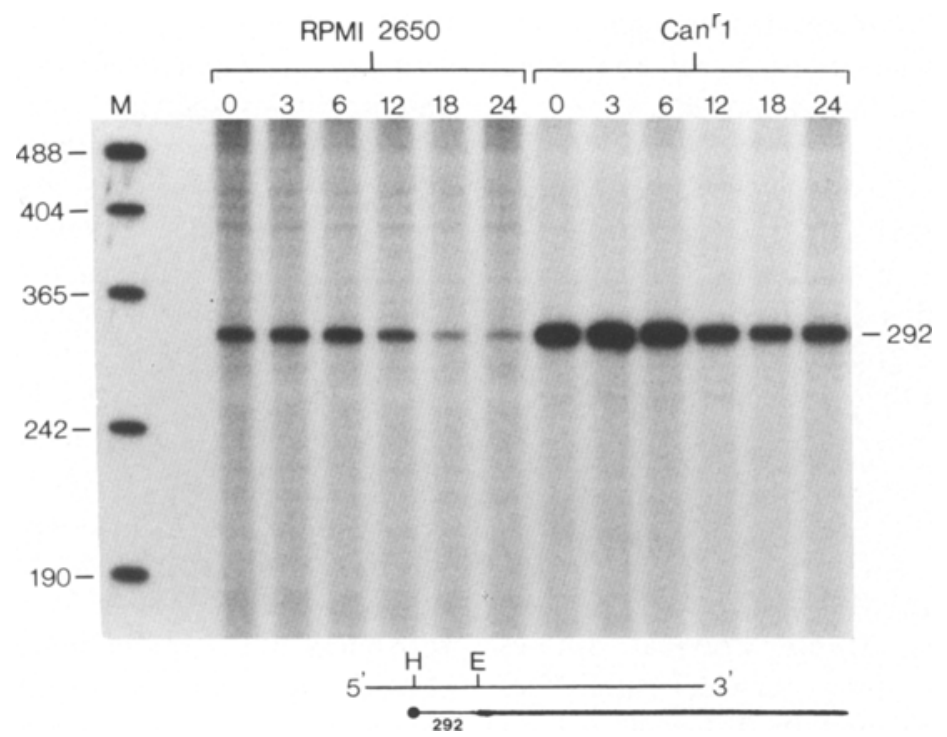

Fig. 1. Estimation of AS mRNA decay rate in parental and canavanine-resistant RPMI 2650 cells. Fifty micrograms of total RNA were used in each assay for RPMI 2650 cells and $5 \mu \mathrm{g}$ were used for Can ${ }^{\mathrm{r}}-1$ cells. The probe was a HindIII-EcoRI restriction fragment of the AS cDNA (thin line, 18) cloned into pUC-8 (thick line). The DNA fragment was linearized at the HindIII site and 3 '-end labeled. The length of the protected region is 292 bases. Numbers above each lane represent hours after addition of actinomycin D. M, radiolabeled markers, pUC- 9 digested with MspI.

nuclear and cytoplasmic components, and AS RNA levels were quantified by $\mathrm{S} 1$ nuclease analysis using either a cDNA (Fig. 2) or intron probe (Fig. 3). The efficiency of the cell fractionation is verified by the complete lack of poly $(\mathrm{A})^{+}$mRNA sequences in the nuclear RNA fraction (Fig. 2, lane $\mathrm{m}$ ) and the relative paucity of intron sequences in the cytoplasmic RNA fraction (Fig. 3, lane e).

The cDNA probe spans several splice junctions and thus protects only fully spliced RNAs. A comparison of total (Fig. 2, lanes a and $\mathrm{d}$ ), cytoplasmic (lanes $\mathrm{b}$ and $\mathrm{e}$ ), and nuclear (lanes $c$ and f) RNA fractions

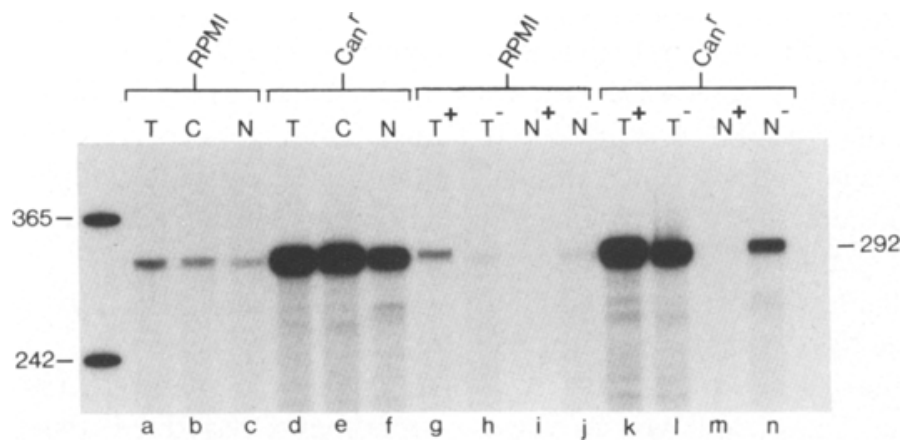

Fig. 2. Cellular fractionation studies quantifying AS RNA levels in RPMI 2650 and Can -1 cells. S1 nuclease assays were performed using the cDNA probe described in Fig. 1. T, total cellular RNA; C, cytoplasmic RNA; N, nuclear RNA. The $(+)$ indicates poly $(\mathrm{A})^{+}$RNA and the $(-)$indicates poly(A) ${ }^{-}$RNA. Fifty micrograms of RNA were used in each assay except for the poly(A) ${ }^{+}$samples in which $5 \mu \mathrm{g}$ were used and in the nuclear poly(A) ${ }^{-}$samples in which $25 \mu \mathrm{g}$ were used. 


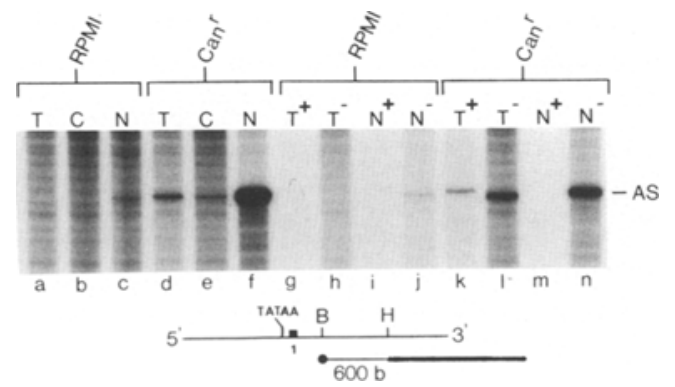

Fig. 3. S1 nuclease assays quantifying AS precursor RNA using an intron 1 probe. Lanes are the same as in Fig. 2. The probe contains a 600-bp BamHI-HindIII restriction fragment of intron 1 of the human $A S$ gene (19) cloned into pUC-8. The probe was linearized at the BamHI site and $3^{\prime}$-end labeled. The protected fragment is approximately $600 \mathrm{bp}$ in length. B, BamHI; H, HindIII. The solid box represents exon 1 in the natural $A S$ gene.

revealed that in each case the amount of AS RNA was increased approximately two orders of magnitude in canavanine-resistant cells. The multiplicity increase is identical when cytoplasmic and nuclear RNA fractions are compared between cell lines, suggesting that efficiency of RNA transport to the cytoplasm was not involved in this regulation. The increased amount of AS RNA in canavanineresistant cell nuclei demonstrates that cytoplasmic mRNA decay is not responsible for increased AS expression in these cells and confirms our conclusion drawn from the mRNA decay experiments (Fig. 1). Both total and nuclear RNA was further fractionated into poly(A) ${ }^{+}$and poly $(\mathrm{A})^{-}$components by chromatography on oligo-dT cellulose, and the level of AS RNA was again quantified in each subfraction by $\mathrm{S} 1$ nuclease analysis. The results show that canavanine-resistant cells also contained greatly increased amounts of nonpolyadenylated AS RNA (Fig. 2, lanes $h$ vs. 1). A smaller yet significant induction of AS RNA was observed in canavanine-resistant cells when nonpolyadenylated nuclear RNA was examined (lanes $j$ vs. n), showing that changes in the concentration of specific RNA in nuclear RNA subpopulations (in this case, fully spliced nuclear nonpolyadenylated RNA) were different than those in the total nuclear RNA pool. Thus, the 200-fold increased level of AS mRNA in canavanineresistant cells is predominantly controlled in the nucleus, and the processes of polyadenylation and RNA transport to the cytoplasm are not involved.

These conclusions were verified by comparison of the amount of AS precursor RNA in parental and canavanine-resistant cells (Fig. 3). Nuclear precursor RNAs containing AS intron sequences were also substantially increased in canavanine-resistant cell nuclei (Fig. 3, lanes c vs. f). Similar findings were obtained using nonpolyadenylated nuclear RNA (lanes $j$ vs. $n$ ). Thus, the mechanism responsible for high expression of AS mRNA in canavanine-resistant cells occurs either at the initiation of transcription (and is not detectable in these cell lines), during elongation of the primary transcript, or at some point prior to RNA polyadenylation and export to the cytoplasm.

Increased Expression of AS Gene in Canavanine-Resistant Cells due to Dominant, Positive-Acting Mechanism. It was of interest to determine whether the increased expression of AS mRNA was due to the gain of a function in canavanine-resistant cells or to the loss of a function in RPMI 2650 cells. The results from such studies would determine whether or not the canavanine-resistant phenotype is dominant and whether genomic DNA transfection would be a feasible means of cloning trans-acting loci that activate $A S$ gene expression. We addressed this question by constructing intraspecific somatic cell hybrids between RPMI 2650 cells and canavanine-resistant cell variants. Distinct dominant selectable markers were introduced into each parental cell line to allow for specific selection of the hybrid progeny following fusion. The dominant selectable marker, RSVneo (5), was introduced into RPMI 2650 cells generating a G418-resistant parental cell line, while the dominant selectable marker, RSVgpt (5), was introduced into the $\mathrm{Can}^{\mathrm{r}}-1$ cell line making this parent resistant to my- 
cophenolic acid. Clones from each parental cell line were fused in the presence of polyethylene glycol and hybrid progeny were selected by growth in media containing G418-HAT/ $\mathrm{M} / \mathrm{X}$ (hypoxanthine, aminopterin, thymidine, mycophenolic acid, xanthine) as outlined in Fig. 4A. Individual clones surviving this double selection were isolated, grown en masse, and DNA and RNA were prepared. Southern blot analysis was used to prove that the selected progeny of the fusion were true hybrids (Fig. 4B). In the example shown, genomic DNA from each parent and three cloned hybrids was digested with EcoRI and probed with RSVcat plasmid sequences. This probe was selected because it detects the integration pattern of both dominant selectable markers and yields distinguishable hybridization patterns for each parent (lanes $a$ and $b$ ). Several of the hybridizing fragments seen in the RPMI 2650 parent are due to an AS promoter-CAT minigene present in this cell line (1). The RPMI 2650 cell parent has three informative bands of $3.2 \mathrm{~kb}, 3.0 \mathrm{~kb}$, and $2.6 \mathrm{~kb}$ (bands 1, 2, and 4) and the Can $\mathrm{Ca}^{\mathrm{r}}-1$ cell parent has one informative band of $2.8 \mathrm{~kb}$ (band 3). The hybridization pattern of each of the three independently isolated hybrids (lanes c-e) was the sum of the parents, confirming that the hybrid cell lines contained genetic information from each parent.

The amount of AS mRNA in each of the three hybrid cell lines was compared to the parental cell lines using $\mathrm{S} 1$ nuclease analysis (Fig. 4C). The results show that in each hybrid cell line, the amount of AS mRNA was approximately equal to the high level found in the canavanine-resistant cell parent. Consistent with this finding is the observation that all of the hybrid cell lines expressed the canavanine-resistant phenotype as demonstrated by their ability to grow in media containing 3 $\mu \mathrm{M}$ canavanine. These findings indicate that high expression of AS mRNA is due to a positive-acting mechanism operating in canavanine-resistant cells and not to the loss of a trans-acting repressor in RPMI 2650 cells.
Consistent with our earlier findings (1), expression of the CAT minigene was not elevated in the hybrid cells when compared to the RPMI 2650 cell parent (data not shown). This observation confirms our previous conclusion that AS promoter-CAT minigenes are not subject to trans-induction in canavanineresistant cells as is the endogenous $A S$ gene.

\section{DISCUSSION}

The goal of these studies was to characterize the molecular basis for high expression of $\mathrm{AS}$ mRNA in canavanine-resistant cell variants. Degradation rates of AS mRNA were estimated in the presence of actinomycin D. In each cell type, the AS mRNA is relatively stable, with a half-life of $12-24 \mathrm{~h}$. These findings show that stabilization of cytoplasmic mRNA is not involved in the induction of AS mRNA in canavanine-resistant cells. Cellular fractionation studies confirm this conclusion. Canavanine-resistant cell nuclei show greatly increased amounts of spliced AS RNA before RNA polyadenylation and transport to the cytoplasm occur. Furthermore, the amount of precursor RNA containing AS intron sequences is greatly increased in canavanine-resistant cell nuclei. Therefore, the control mechanism must act very early in the synthesis or processing of AS precursor RNA. An abundant amount of nonpolyadenylatedspliced AS RNA was detected in this study (Fig. 2). This suggests that splicing of the AS primary transcript may precede polyadenylation, although inefficient fractionation could also give rise to these results (due to short poly(A) tails). Our observations are in contrast to previous studies that examined the processes of splicing and polyadenylation in the SV40 late gene RNAs and adenovirus 2 RNAs $(11,12)$. Those studies concluded that polyadenylation precedes splicing. The significance of this observation is unclear at present and is currently being investigated.

We constructed intraspecific somatic cell hybrids between RPMI 2650 cells, which 

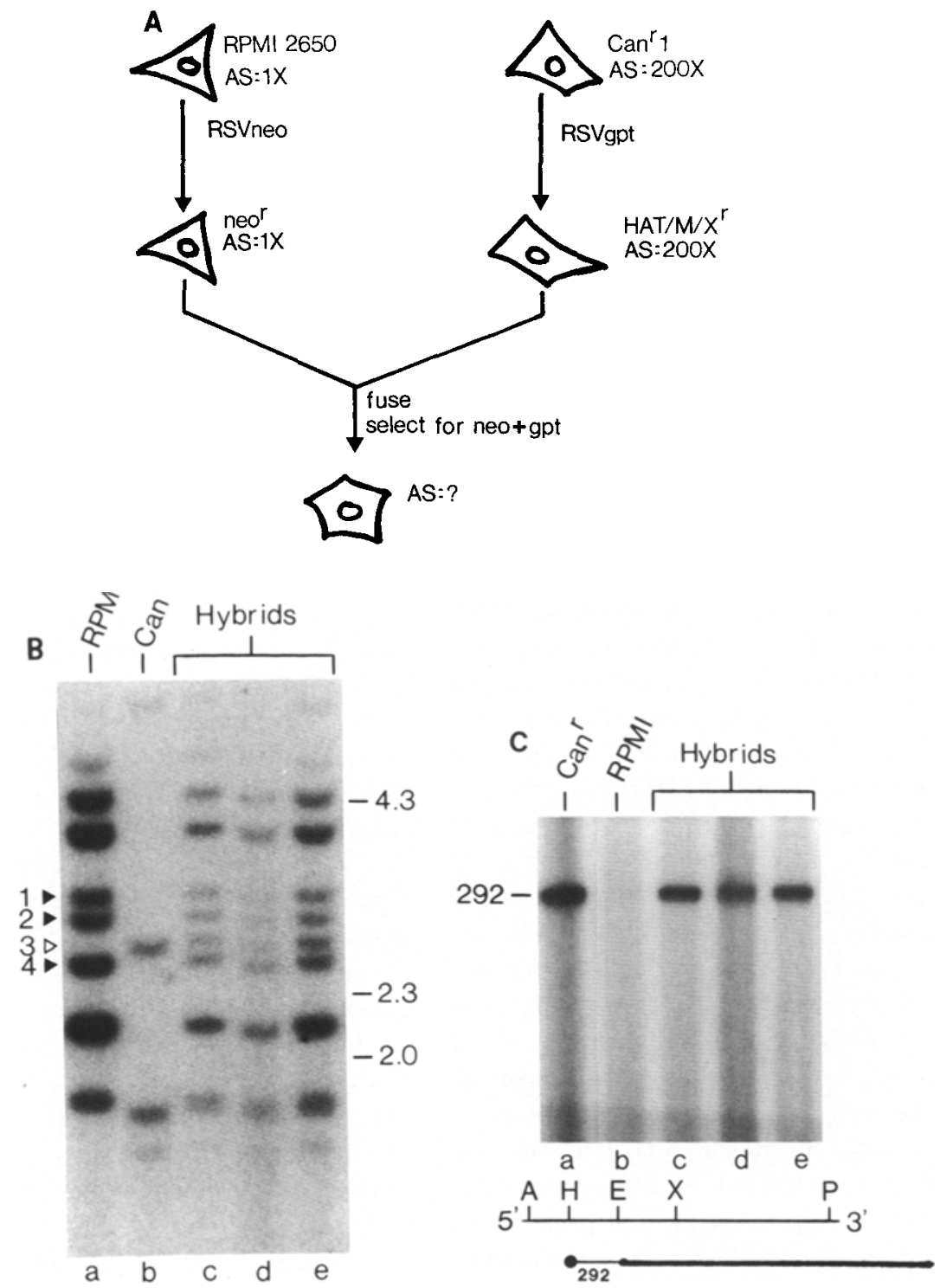

Fig. 4. (A) Strategy for construction of somatic cell hybrids testing for dominance. (B) Southern blot pattern of parental and hybrid cell lines. Ten micrograms of genomic DNA from each cell line were digested with EcoRI and subjected to Southern blot analysis. The probe was radiolabeled RSVcat (20) plasmid DNA. Lane a, RPMI 2650 parent containing an AS promoter-CAT minigene, pmgAS-10, and RSVneo (1); lane b, Can ${ }^{r}-1$ parent containing RSVgpt; lanes c-e, cloned hybrid cell lines C6, B1, and C1. (C) S1 nuclease assays quantifying AS mRNA expression in parental and hybrid cell lines. Fifty micrograms of total RNA were used in each assay. The lanes are the same as in B. The probe was the same as in Fig. 1. Below the autoradiogram is a partial restriction map of the AS cDNA. A, AvaI; H, HindIII; E, EcoRI, X, Xhol; P, PvuII. 
express a low level of AS mRNA, and canavanine-resistant cell variants, which express a high level, to determine whether high expression of AS mRNA is dominant or recessive. All hybrid cell lines contain a high level of AS mRNA, implicating a positive-acting mechanism operating in the canavanine-resistant cell. These experiments rule out the possibility that the induction of AS mRNA in canavanine-resistant cells is due to the loss of a trans-acting repressor. This assumes that no specific loss of chromosomes occurred in the human-human cell hybrids. It is important to note that the cell fusion studies do not discriminate between a cis- or trans-acting mechanism. However, studies using canavanineresistant human lymphoblasts have indicated that the induction of AS mRNA in canavanine-resistant cell variants is due to a transacting mechanism (1). We feel that the mechanism by which mammalian cells induce $A S$ gene expression in canavanine-resistant variants is likely to be common among different cell types. Consistent with this idea are the observations that the multiplicity of induction of AS mRNA in canavanine-resistant lymphoblasts and RPMI 2650 cells is similar (200 to 300 -fold) $(4,13)$.

Two questions remain unanswered regarding the mechanism underlying high AS mRNA expression in canavanine-resistant cell variants: (1) whether the mechanism is acting at the transcriptional or nuclear posttranscriptional level and (2) whether the mechanism is activated in the canavanineresistant cell by a genetic or epigenetic event. If induction of AS mRNA expression is transcriptional, the cis-acting element necessary for this regulation must exert its effect through a great distance. We base this statement on the findings that AS minigenes containing genomic sequences $3 \mathrm{~kb} 5^{\prime}$ and $12 \mathrm{~kb} 3^{\prime}$ from the transcription initiation site fail to express at higher levels in canavanine-resistant cells as compared to parental RPMI 2650 cells $(1,2)$. Furthermore, if the cis-acting element is intragenic, it must lie in an intron since AS cDNA minigenes also fail to express at higher levels in canavanine-resistant cell variants (21). It is clear, however, that if the mechanism is transcriptional, it must involve sequences other than those present in the immediate $5^{\prime}$ flanking region of the $A S$ gene. All of our data concerning induction of the $A S$ gene in canavanine-resistant cells are consistent with a nuclear posttranscriptional mechanism. Posttranscriptional control has been implicated in the regulation of several genes that are expressed abundantly in liver similar to AS $(9,14-16)$. These include genes that are expressed exclusively in liver (albumin, phenylalanine hydroxylase, adolase $B$, transferrin) and those expressed at a high level in liver and at low levels in most other nonhepatic tissues (phosphoenolpyruvate carboxykinase). Thus, induction of $A S$ gene expression in canavanine-resistant cells shares at least one characteristic with liver-specific gene expression in that high cytoplasmic mRNA levels are achieved through a positiveacting nuclear mechanism.

Several observations suggest that the mechanism underlying induction of the $A S$ gene is activated epigenetically in canavanineresistant cells. For instance, the frequency of obtaining canavanine-resistant cell variants is not increased when cells are treated with point mutagens (ethylmethyl sulfonate), agents that alter DNA methylation (5-azacytidine), or agents that promote gene amplification (tumor promoters and hydroxyurea, unpublished data). These observations suggest, but do not prove, that the mechanism underlying high $A S$ expression is not activated through covalent changes in DNA. They also argue against the possibility that the $A S$ gene is transcriptionally activated in the canavanineresistant cell by a cis-acting mutation (such as a base change in the AS promoter region). Interestingly, our observations are consistent with those made with dedifferentiated variants of rat hepatoma cells that spontaneously 
reacquire a liver cell-like phenotype in culture (17). Preliminary experiments have shown that the frequency of isolating canavanineresistant cell variants increases 20 - to 50 -fold above the background frequency following genomic DNA transfection (R. Pogulis and S. Freytag, unpublished data). The increase in frequency is identical when either RPMI 2650 cell or canavanine-resistant cell genomic DNA is used but is substantially higher than mock-transfected cells. Taken together, these observations suggest that activation of $A S$ gene expression is not the result of a genetic change in canavanine-resistant cells, but instead is consistent with an epigenetic mechanism. Since it is likely that cell differentiation is controlled epigenetically, we feel these observations support our contention that the canavanine-resistant cell variant represents a more differentiated cell type that has activated a mechanism normally used in cell differentiation, perhaps one used during liver differentiation. Given that the canavanineresistant phenotype is selectable and the knowledge that the phenotype is dominant, this system provides a powerful means of isolating trans-acting loci that induce a differentiated cell trait.

\section{ACKNOWLEDGMENTS}

We thank Mark Anderson for helpful discussions and Len Lutter and Mike Imperiale for critical readings of the manuscript. F.M.B. has been supported by the Burton Baker Memorial Fellowship from the Michigan Cancer Research Center, and training grants in Cellular and Molecular Biology and the Medical Scientist Training Program. This research was supported by grants from the
National Institutes of Health and the American Cancer Society.

\section{LITERATURE CITED}

1. Boyce, F., Anderson, G., Rusk, C., and Freytag, S. (1986). Mol. Cell. Biol. 6:1244-1252.

2. Jackson, M., O'Brien, W., and Beaudet, A. (1986). Mol. Cell. Biol. 6:2257-2261.

3. Irr, J., and Jacoby, L. (1978). Somat. Cell Genet. 4:111-124.

4. Su, T.-S., Bock, H.-G., O'Brien, W., and Beaudet, A. (1981). J. Biol. Chem. 256:11826-11831.

5. Gorman, C., Padmanabhan, R., and Howard, B. (1983). Science 221:551-553.

6. Maniatis, T., Fritsch, E., and Sambrook, J. (1982). Molecular cloning: A laboratory manual, (Cold Spring Harbor Press, Cold Spring Harbor, New York).

7. Weaver, R., and Weissman, C. (1979), Nucleic Acids Res. 7:1175-1193.

8. McKnight, G., and Palmiter, R. (1979). J. Biol. Chem. 254:9050-9058.

9. Clayton, D., Weiss, M., and Darnell. J. (1985). Mol. Cell. Biol. 5:2633-2641.

10. Leys, E., Crouse, G., and Kellems, R. (1984). J. Cell. Biol. 99:180-187.

11. Lai, C., Dhar, R., and Khoury, G. (1978). Cell 14:971-982.

12. Nevins, J., and Darnell, J. (1978). Cell 15:14771493.

13. Jacoby, L. (1978). Somat. Cell Genet. 4:221-231.

14. Sala-Trepat, J., Poiret, M., Sellem, C., Bessada, R., Erdos, T., and Gluecksohn-Waelsch, S. (1985). Proc. Natl. Acad. Sci. U.S.A. 82:2442-2446.

15. Kazmaier, M., Bruning, E., and Ryffel, G. (1985). EMBO J. 4:1261-1266.

16. Loose, D., Shaw, P., Krauter, K., Robinson, C., Englard, S., Hanson, R., and Gluecksohn-Waelsch, S. (1986). Proc. Natl. Acad. Sci. U.S.A. 83:51845188 .

17. Deschatrette, L., Moore, E., Dubois, M., and Weiss, M. (1980). Cell 19:1043-1051.

18. Bock, H.-G., Su, T.-S., O'Brien, W., and Beaudet, A. (1983). Nucleic Acids Res. 11:6505-6512.

19. Freytag, S., Beaudet, A., Bock, H.-G., and O'Brien, W. (1984). Mol. Cell. Biol. 4:1978-1984.

20. Gorman, C., Merlino, G., Willingham, M., Pastan, I., and Howard, B. (1982). Proc. Natl. Acad. Sci. U.S.A. 79:6777-6781.

21. Boyce, F., Pogulis, R., and Freytag, S. (1989). Somat. Cell Mol. Genet. 15:123-127. 